\title{
A Simple Mnemonic for Differential Diagnosis of Prolonged Oxygen or Respiratory Support Dependence in COVID-19 Patients
}

\author{
Sugata Dasgupta ${ }^{1}$, Atanu Chandra ${ }^{2}$ \\ Bengal Physician Journal (2021): 10.5005/jp-journals-10070-7060
}

The second wave of the novel coronavirus disease-2019 (COVID-19) in India has been more devastating than the first, in terms of a large number of caseloads, spread of infections in the younger population, and increased severity, thereby creating a huge pressure on the healthcare systems. The disease pattern is also different with quite a few patients needing prolonged oxygen or respiratory support. ${ }^{1}$ Some are getting into this prolonged support-dependent phase even after showing initial signs of improvement. Due to huge caseloads, both primary care physicians and doctors across all specialties (including residents) have to deal with such patients, even in resource-limited areas, where immediate investigations are not always possible. Moreover, transport of support-dependent patients to radiology suits for computer tomography (CT) is also being impossible even in good setups. In this scenario, we would like to propose a simple mnemonic (PE IF AF: pneumonia: non-resolved/ organizing; embolus; infection; fibrosis; airspaces; fluid and failure), so that even junior doctors and doctors across specialties with no formal training in acute medicine (but who have been deployed in acute care due to the pressure exerted by the pandemic on the healthcare system) can approach prolonged oxygen or respiratory support-dependent patients easily in a systematic way, without missing anything (Box 1).

Box 1: Mnemonic for differential diagnosis of prolonged oxygen or respiratory support dependence in patients with COVID-19

\begin{tabular}{|ll|}
\hline P & Pneumonia (non-resolved/organizing) \\
E & Embolus \\
I & Infection (secondary bacterial or fungal) \\
F & Fibrosis \\
A & Airspaces such as pneumothorax, pneumatocele, or cavities \\
F & Failure (cardiac) and fluid (pleural effusion or pulmonary edema) \\
\hline
\end{tabular}

Pneumonia (non-resolved/organizing) [P]: The classical radiological feature of COVID-19 related pneumonia is ground glass opacity (GGO), which is mainly distributed in peripheral and subpleural locations. A sizable proportion of patients with COVID-19 pneumonia have persistent radiological abnormalities probably due to ongoing inflammatory activity, even after a few weeks after discharge and many of them require long-term. Clinico-radilogically confirmed patients of COVID-19 pneumonia should be followed-up at least three weeks after diagnosis to recognize early lung damage; in the subset of patients having post-COVID-19 organizing pneumonia, glucocorticoids are effective treatment option. ${ }^{2}$
1Department of Critical Care Medicine, RG Kar Medical College and
Hospital, Kolkata, West Bengal, India
${ }^{2}$ Department of Internal Medicine, RG Kar Medical College and
Hospital, Kolkata, West Bengal, India

Corresponding Author: Atanu Chandra, Department of Internal Medicine, RG Kar Medical College and Hospital, Kolkata, West Bengal, India, Phone: +91 033-25304557; +91 9474190374, e-mail: chandraatanu123@gmail.com

How to cite this article: Dasgupta S, Chandra A. A Simple Mnemonic for Differential Diagnosis of Prolonged Oxygen or Respiratory Support Dependence in COVID-19 Patients. Bengal Physician Journal 2021;8(3):76-77.

Source of support: Nil

Conflict of interest: None

Embolus [E]: Thrombosis in the pulmonary vasculature is a very important cause of clinical worsening and increasing oxygen requirements in patients with COVID-19, which may be attributed to the combined effect of the severe inflammatory response along with endothelial damage. ${ }^{3} \mathrm{~A}$ contrast-enhanced CT scan of thorax along with CT-pulmonary angiography should be performed in where the refractory hypoxia cannot be explained by extension of lung parenchymal involvement alone. A marked elevation of D-dimer levels should raise our suspicion in such patients.

Infection (secondary bacterial or fungal) [I]: Secondary bacterial and fungal infection is a very important cause of clinical deterioration and worsening oxygenation mainly in critically ill patients, especially in those receiving immunosuppressants. While most of the respiratory bacterial infections reported in patients with COVID-19 are caused by Streptococcus pneumonia and Staphylococcus aureus; majority of the hospital-acquired pneumonia and bacteremia are seen to be associated with Pseudomonas aeruginosa, Klebsiella, Escherichia coli, and Staphylococcus aureus. ${ }^{4}$ Among the fungal infections, Aspergillus fumigates, Pneumocystisjirovecii, and recently several cases COVID-19 associated mucormycosis (CAM) are being reported worldwide. Therefore, appropriate microbiological investigations (mainly sputum culture) and judicious use of antibiotic/antifungal agents are of utmost importance in such patients. ${ }^{5}$

Fibrosis [F]: Progressive pulmonary fibrosis is one of the most worrying consequences of COVID-19 pneumonia in which there is irreversible distortion of pulmonary architecture and those 
patients usually present with shortness of breath, dry cough, and persistent oxygen desaturation. ${ }^{6}$ Older age, male sex, smoking, need for mechanical ventilation, longer duration of hospitalization or intensive care unit stay, more severity of disease, and development of acute respiratory distress syndrome (ARDS) are the risk factors for post COVID-19 lung fibrosis.

Airspaces such as pneumothorax, pneumatocele, or cavities $[A]$ : Pneumatocele or pneumothorax often develops in COVID-19 pneumonia commonly as a complication, under positive pressure ventilation or in association with preexisting lung conditions; they are often responsible for refractory hypoxemia. ${ }^{7}$

Failure (cardiac) and fluid (pleural effusion or pulmonary edema) [F]: Heart failure may be encountered de novo or in patients with preexisting cardiac disease, due to several mechanisms such as hyperinflammatory state, direct cardiac injury, endothelial damage, stress cardiomyopathy, and pulmonary hypertension leading to right heart failure. ${ }^{8}$ Pleural effusion in the background of COVID-19 may occur due to multiple factors such as heart failure, increased interstitial edema, and capillary permeability, and it is associated with poorer outcome.

We think, remembering this simple mnemonic may help the physicians to appropriately tailor the clinical evaluation, investigations, management and prognosticate outcomes in patients with COVID-19 requiring prolonged oxygen, or respiratory support dependence, especially in resource-limited settings.

\section{Contributorship Statement}

Both the authors contributed to conception and design, analysis and interpretation, critical revision of content, and final approval of manuscript. Both the authors are in agreement to be accountable for all aspects of the work in ensuring that questions related to the accuracy or integrity of any part of the work are appropriately investigated and resolved.

\section{References}

1. Daher A, Balfanz P, Aetou M, et al. Clinical course of COVID-19 patients needing supplemental oxygen outside the intensive care unit. Sci Rep 2021;11(1):2256. DOI: 10.1038/s41598-021-81444-9. PMID: 33500431 ; PMCID: PMC7838409.

2. George PM, Barratt SL, Condliffe R, et al. Respiratory follow-up of patients with COVID-19 pneumonia. Thorax 2020;75(11):1009-1016. DOI: 10.1136/thoraxjnl-2020-215314. PMID: 32839287; PMCID: PMC7447111.

3. Chandra A, Chakraborty U, Ghosh S, et al. Anticoagulation in COVID-19: current concepts and controversies. Postgrad Med J 2021:postgradmedj-2021-139923. DOI: 10.1136/postgradmedj2021-139923. PMID: 33850011.

4. Rawson TM, Wilson RC, Holmes A. Understanding the role of bacterial and fungal infection in COVID-19. Clin Microbiol Infect 2021;27(1):9-11. DOI: 10.1016/j.cmi.2020.09.025. PMID: 32979569; PMCID: PMC7546203.

5. Falcone M, Tiseo G, Giordano C, et al. Pisa COVID-19 Study Group. Predictors of hospital-acquired bacterial and fungal superinfections in COVID-19: a prospective observational study. J Antimicrob Chemother 2021;76(4):1078-1084. DOI: 10.1093/jac/dkaa530. PMID: 33374002; PMCID: PMC7799007.

6. Ali RMM, Ghonimy MBI. Post-COVID-19 pneumonia lung fibrosis: a worrisome sequelae in surviving patients. Egypt J Radiol Nucl Med 2021;52(1):101. DOI: 10.1186/s43055-021-00484-3. PMCID: PMC8047597.

7. Martinelli AW, Ingle T, Newman J, et al. COVID-19 and pneumothorax: a multicentre retrospective case series. Eur Respir J 2020;56(5): 2002697. DOI: 10.1183/13993003.02697-2020.

8. Rey JR, Caro-Codón J, Rosillo SO, et al. Heart failure in COVID-19 patients: prevalence, incidence and prognostic implications. Eur J Heart Fail 2020;22(12):2205-2215. DOI: 10.1002/ejhf.1990. PMID: 32833283; PMCID: PMC7461427. 